\title{
Evaluation of the Effect of Mycotoxins in Naturally Contaminated Feed on the Efficacy of Preventive Vaccine against Coccidiosis in Broiler Chickens
}

\author{
Anwaar M. Elnabarawy ${ }^{1 *}$, Marwa M. Khalifa ${ }^{2}$ Khaled S.Shaban $^{1}$ and Walied S. Kotb ${ }^{3}$ \\ ${ }^{I}$ Department of poultry diseases, Faculty of Veterinary Medicine, Cairo University, Giza, Egypt \\ ${ }^{2}$ Department of Parasitology, Faculty of Veterinary Medicine, Cairo University, Giza, Egypt \\ ${ }^{3}$ Department of Pathology, Faculty of Veterinary Medicine, Kafr el-sheikh University, Egypt \\ *Corresponding author's Email: anwaar.elnabarawy@gmail.com; ORCID: 0000-0003-1618-6842
}

\begin{abstract}
This research was designed to evaluate the effect of naturally contaminated feed with mycotoxins on the efficacy of vaccination against coccidiosis in broilers. Two hundred day-old Hubbard broiler chicks were divided into four groups (50 chicks/group). Group 1 and 3 were kept on naturally contaminated diets containing 4 ppb aflatoxin, 3 ppb ochratoxin, $1 \mathrm{ppm}$ zearalenone and $2 \mathrm{ppb}$ aflatoxin, $6 \mathrm{ppb}$ ochratoxin and $1 \mathrm{ppm}$ zearalenone in starter and grower feed, respectively. Groups 2 and 4 were fed on diet without detectable levels of mycotoxins. Group 1 and 2 were vaccinated with anticoccidial vaccine at 4 days of age. All groups were challenged with Eimeria tenella $\left(5 \times 10^{4} /\right.$ chick) 14 days post-vaccination. Vaccinated mycotoxicated birds showed a significant reduction in body weight, high mortality, significant oocysts shedding, severe hemorrhagic typhlitis, marked lymphoid depletion in bursa of Fabricius and degenerative changes in liver and kidney. In addition, a remarkable decrease in length and width of intestinal villi, mucosal length and crypt depth. Feed contamination with multi-mycotoxins in permissible level caused vaccination failure and a remarkable decrease in intestinal morphometric histopathological parameters.
\end{abstract}

Received: 18 Feb. 2020

Accepted: 29 Mar. 2020

Key words: Coccidia Vaccine, Mycotoxins, Poultry Feed.

\section{INTRODUCTION}

Mycotoxicosis was firstly described by Forgacs and Carll (1955) as toxicosis arising from fungus-infested feed. They reported a hemorrhagic condition in poultry which was associated with the ingestion of fungus and fungal products in moldy feed. Later in the early 1960, an acute hepatotoxic disease epidemic struck the turkey population in England.

The presence of multiple mycotoxins simultaneously in feed commonly occur as a result of the presence of many fungal species in feed producing several different mycotoxins simultaneously or due to formation of poultry feed from different feed ingredient with different sources, each of which is contaminated with a different mycotoxin (Trenholm et al., 1989). The interactive effects of mycotoxins, when occur in combinations, may be synergistic, potentiated, or even antagonistic (Kubena et al., 1988).

The most common clinical signs of mycotoxicosis in broiler chickens are reduced feed intake, weight gain, poor food conversion ratio, increased mortality and reduced immune responses including poor antibody titer and lymphoid organ damage subsequently increased susceptibility to different infections (Rosa and Santurio, 2005; EFSA, 2009; Resanovic et al., 2009).

Immunosuppressive effects of mycotoxins are due to effect on serum proteins, macrophages, complement and interferon are because of inhibition of protein synthesis and liver damages (Resanovic et al., 2009). Mycotoxins also cause aplasia of bursa of Fabricius, thymus, and spleen in chicken, which results in a marked decrease in cellular and antibody responsiveness of immune system (Karaman et al., 2005). Moreover, mycotoxins induced marked morphological alteration in intestinal histology.

The most common pathogenic Eimeria species affecting chickens are Eimeria necatrix, $E$ tenella, $E$. acervulina, E. maxima, and E. brunetti. Infection with Eimeria spp. causes chicken coccidiosis that leads to mortality, decreased weight gain and weights uniformity of birds flock (McDougald and Fitz-Coy, 2013). This protozoal disease causes enormous economic losses with a global impact estimated to be over 3 billion USD per year 
in the poultry industry (Dalloul and Lillehoj, 2006). Moreover, coccidiosis is considered an important factor for the development of clostridial infection particularly necrotic enteritis (Dahiya et al., 2006; Collier et al., 2008).

Poultry field protecting different poultry species and performance from coccidiosis challenge by acquired immunity (Shirley et al., 1995). So control of coccidiosis is achieved by vaccination as an alternative to chemotherapy as it is overcoming the problem of drug resistant resulting from usage of chemotherapy. Anticoccidial vaccines are composed of live oocysts of attenuated or non-attenuated strains of Eimeria (Shirley et al., 2007). Therefore, this study aimed to evaluate the effect of mycotoxins determined in naturally contaminated broilers feed and proven to be within the permissible levels, on the efficacy of vaccines recommended against coccidiosis through different parameters.

\section{MATERIAL AND METHODS}

\section{Ethical approval}

This study was approved by Institutional Animal Care and Use Committee (IACUC), Cairo University (VetCU1010201903).

\section{Broilers feed}

Commercial feed specified for broilers was analyzed for detection and determination of contamination levels for three important mycotoxins (aflatoxin, ochratoxin, and zearalenone) in starter and grower feed types. Where feed bags were thoroughly mixed to obtain representative feed samples and fluorometer series 4 and protocol of manual were used for quantitive determination of aflatoxin, ochratoxin, and zearalenone according to AOAC (1995) and FAO (2003).

\section{Chicks}

Two hundred day-old Hubbard broiler chicks were employed in this experiment.

\section{Coccidial oocysts}

\section{Collection and sporulation of oocysts}

Eimeria species oocysts used for challenge were collected from ceci of dead naturally infected chickens. The collected oocysts were cleaned and incubated for 48 hours in $2.5 \%$ potassium dichromate $\left(\mathrm{K}_{2} \mathrm{Cr}_{2} \mathrm{O}_{7}\right)$ solution for sporulation according to Khaier et al. (2015).

\section{Purification, identification and counting of oocysts}

Purification of sporulated oocysts was done according to Khaier et al. (2015). The sporulated oocysts were mainly identified as Eimeria tenella according to its confined caecal part in naturally infected chickens and due to its typical measurements of E. tenella according to Khaier et al. (2015). Counting E. tenella oocysts was done using McMaster Technique according to Soulsby (1982).

\section{Infection and challenge}

After count of sporulated oocysts (3 replicate), the oocysts were allocated in separate doses each of $5 \times 10^{4}$. Ten Birds from vaccinated and non-vaccinated groups were inoculated intra-croup using suitable rubber syringe at the recommended day of challenge (Velkers et al., 2010). Oocysts output in feces of challenged birds were evaluated weekly until the end of experiment, pooled samples from dropping of the inoculated birds were collected (from each group) as before.

\section{Sample collection}

Fecal samples (droppings) were collected weekly from all groups.

\section{Histopathological samples}

Tissue samples from intestine, bursa of Fabricius, liver, and kidney were collected weekly from different groups. These samples were fixed in $10 \%$ neutral buffered formalin, sectioned at 5-6 $\mu \mathrm{m}$ thicknesses and stained with Hematoxylin and Eosin (H\&E) stain (Bancroft et al., 1996).

\section{Evaluation of parameters}

Chicks were monitored daily for clinical symptoms, mortality and post-mortem lesions. Counting of sheded oocyst in both vaccinated, nonvaccinated groups. Body weight, intestinal lesion scoring of morphometric histopathological lesions with different treated groups were recorded. Challenging parameters (mortality \% and oocyst shedding) were calculated.

\section{Experimental design and housing}

The experiment was conducted in the poultry experimental units of Poultry Diseases Department, Faculty of Veterinary Medicine, Cairo University, after cleaning and disinfection. Two hundred day-old Hubbard broiler chicks and commercial diet specified for broilers feeding free from anticoccidial and antimycotoxins were employed in this study. The chicks were divided into four groups (50 chicks/group). Group 1 and 3 (control positive groups) were kept on naturally contaminated diet 
containing $4 \mathrm{ppb}, 3 \mathrm{ppb}, 1 \mathrm{ppm}$ and $2 \mathrm{ppb}, 6 \mathrm{ppb}$ and 1 ppm aflatoxin, ochratoxin and zearalenone in starter and grower feed, respectively. GroupS 2 and 4 (control negative groups) were fed on mycotoxins free diet. Groups 1 and 2 were vaccinated at age $4^{\text {th }}$ day with anticoccidial vaccine via eye instillation. Group 3, $4 \mathrm{kept}$ as control positive and control negative non vaccinated groups. Appropriate temperature, humidity, feeding, and lighting program were followed according to standard recommended by supplies. At $14^{\text {th }}$ day post-vaccination, 10 chicks from each group (1-4) were challenged with $5 \times 10^{4}$ live Eimeria tenella sporulated oocysts. All birds were vaccinated at 7 days of age with Hitchner B via eye instillation, at 10 days of age with inactivated avian influenza and Newcastle disease virus (NDV) via subcutaneous route at a dose of $0.5 \mathrm{ml}$ per bird. At 13 days of age, birds were vaccinated with live intermediate Gumboro strain via eye instillation. Finally, all birds were vaccinated by the NDV Lasota vaccine at 20 days of age via eye instillation.

\section{Statistical analysis}

PASW Statistics, SPSS 18.0 software (SPSS Inc., Chicago, IL, USA) was used to analyze the data. Two-way ANOVA was used to compare means between different groups. Differences were considered statistically significant at $P$-value $<0.05$.

\section{RESULTS}

\section{Clinical signs}

Mycotoxicated groups (G1 and G3) showed ununiform growth pattern, whitish droppings, lameness and inability to stand. Moreover, diarrhea tinged with blood was recorded on 8-10 days post-vaccination in group 1 (control positive mycotoxicated vaccinated group). The mortality rate was $40 \%$ in mycotoxicated vaccinated group.

\section{Postmortem lesions}

Retardation in growth, severe hemorrhagic typhlitis observed in control positive (mycotoxicated vaccinated group) compared to few petechial hemorrhages on cecum of control negative vaccinated group (Figures 1 and 2). Petechial hemorrhages and grayish-white foci (schizogony) indicating Eimeria necatrix infection in control positive (mycotoxicated vaccinated group 1) (Figure 3). In addition to severe hemorrhagic typhlitis observed in control positive vaccinated challenged group compared to mild hemorrhagic typhlitis in vaccinated challenged control negative group (Figure 4). Vaccinated challenged control negative group showed mild hemorrhagic typhlitis. Vaccinated challenged mycotoxicated group (control positive) showed severe hemorrhagic typhlitis and challenged control negative non-vaccinated group showed inspciated hemorrhagic typhlitis (Figure 5). Moreover, pale yellow liver, marked lobulation and paleness in kidney were constant macroscopic lesions recorded in mycotoxicated groups during the experimental period.

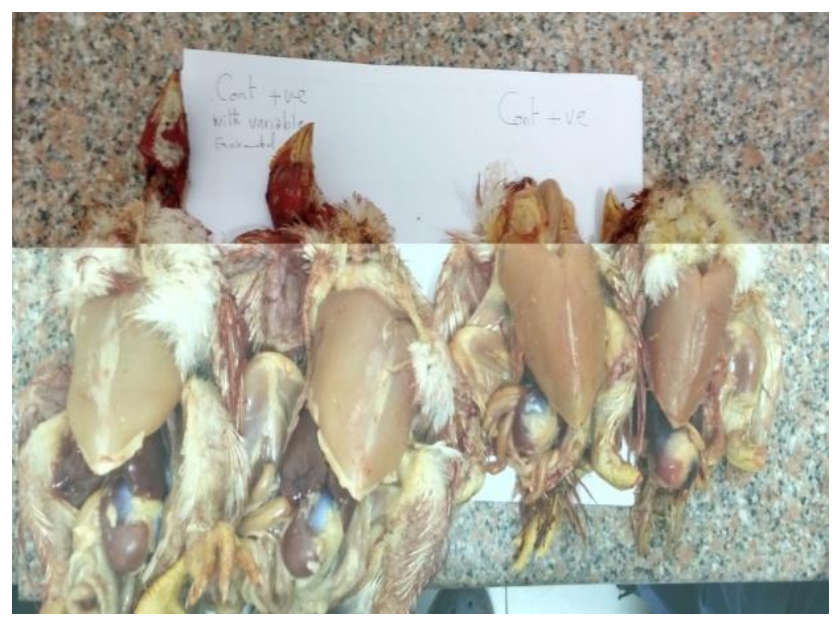

Figure 1. Reduction in body weight gain recorded in broiler chicks fed on mycotoxin naturally contaminated feed and vaccinated with anticoccidial vaccine (right) compared to normal growth pattern in negative control vaccinated group (left).

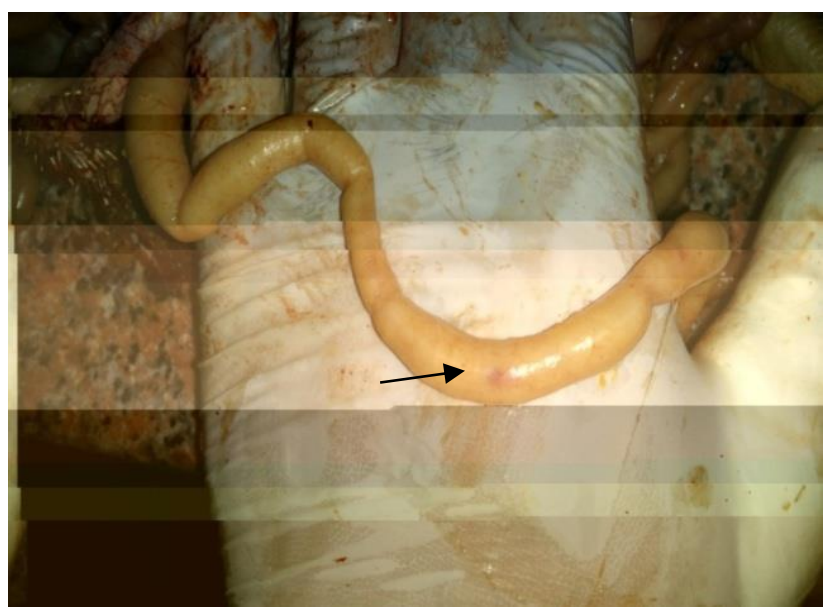

Figure 3. Petechial hemorrhages and grayish-white foci (schizogony) indicating Eimeria necatrix infection in broiler chick fed on mycotoxin contaminated feed and vaccinated with anticoccidial vaccine. 


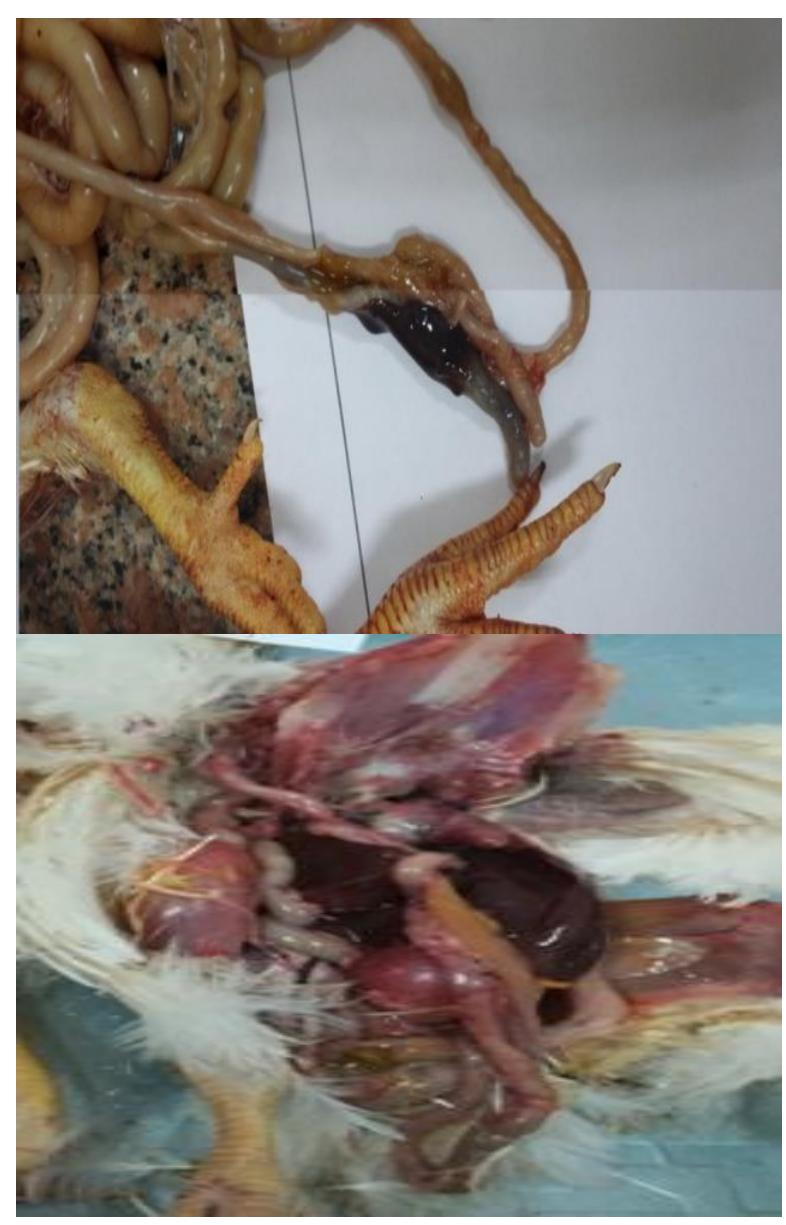

Figure 2. Severe hemorrhagic typhlitis observed in 14-day old broiler chicks fed on mycotoxin-contaminated feed and vaccinated with anticoccidial vaccine (up) compared to few petechial hemorrhages on cecum of negative control vaccinated birds (down).
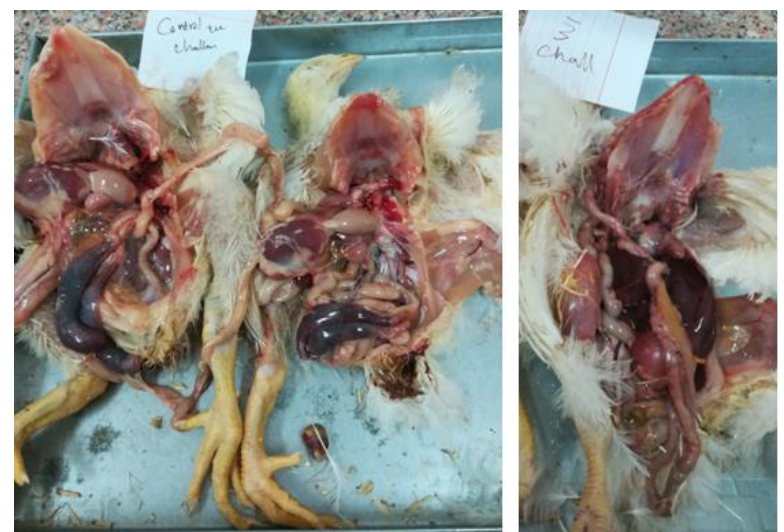

Figure 4. Severe hemorrhagic typhlitis was observed in birds fed on mycotoxin contaminated feed and vaccinated with anticoccidial vaccine and challenged with Eimera tenella oocysts $\left(5 \times 10^{4} / \mathrm{chick}\right) 14$ days post vaccination (left). Mild hemorrhagic typhlitis was observed in birds fed on mycotoxin free diet, vaccinated with anticoccidial vaccine and challenged with Eimera tenella oocysts $\left(5 \times 10^{4} /\right.$ chick) 14 days post vaccination (right).

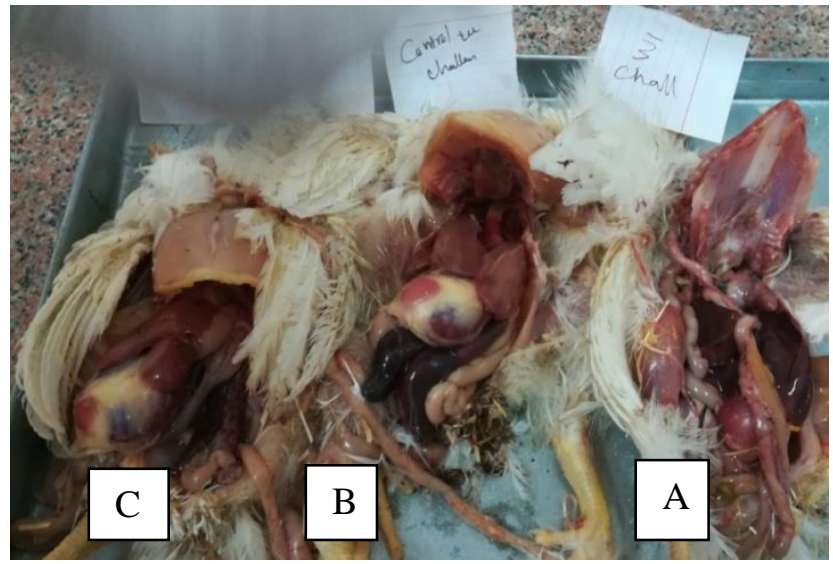

Figure 5. Post-mortem examination of broilers chickens challenged with Eimera tenella oocysts $\left(5 \times 10^{4} /\right.$ chick $) 14$ days post-vaccination. (A) Mild hemorrhagic typhlitis recorded in bird vaccinated with anticoccidial vaccine and kept on mycotoxins free diet. (B) Severe hemorrhagic typhlitis observed in bird vaccinated with anticoccidial vaccine and fed with mycotoxins contaminated feed. (C) Inspcisated hemorrhagic typhlitis observed in nonvaccinated bird fed with mycotoxins free diet.

\section{Body weight, Eimeria oocysts count}

The mycotoxicated groups either vaccinated or nonvaccinated (G 1 and G3) statistically recorded a significant reduction in body weight compared to mycotoxins free control negative groups (G 2 and G4) as showed in table 1 .

Table 2 presents that mean coccidial oocysts count shed from mycotoxicated vaccinated group significantly higher than those shed from control negative vaccinated group. In control negative and control positive nonvaccinated groups (G3 and G4), none of oocysts were detected along the experimental time. A two-way analysis of variance yielded a main effect for the groups, $F(1,16)$ $=39.963, p<0.0001$, the oocyst shedding was significantly higher for group $1\left(\mathrm{M}=2.8 \times 10^{6}, \mathrm{SE}=4.9 \times 10^{5}\right)$ than for group $2\left(\mathrm{M}=1.5 \times 10^{6}, \mathrm{SE}=3.0 \times 10^{5}\right)$. The main effect of weeks was significant, $F(3,16)=45.027, p<0.0001$. Moreover, the interaction effect was significant, $F(3,16)$ $=3.395, p=0.044$, There was a statistically significant interaction between the effects of groups and weeks on the shedding of coccidial oocyst.

Table 3 showing oocyst coccidial count shed from vaccinated challenged and non-vaccinated challenged groups significantly higher number in group 1, 3 than group 2, 4. A two-way analysis of variance yielded a main effect for the groups, $F(3,16)=12.228, p<0.0001$, the oocyst shedding was significantly lower for group 2 $\left(\mathrm{M}=1.0 \times 10^{6}, \mathrm{SE}=3.9 \times 10^{5}\right)$ than for groups 1,3 and 4 . The main effect of weeks was significant, $F(1,16)=280.688$, $p<0.0001$. Moreover, the interaction effect was significant, 
$F(3,16)=4.184, p=0.023$, There was a statistically significant interaction between the effects of groups and weeks on the shedding of coccidial oocysts.

\section{4-Mortality pattern}

Mortality rate recorded in different groups was $40 \%$ in control positive vaccinated group, $0 \%$ in control negative vaccinated group and control negative vaccinated challenged, $60 \%$ in vaccinated challenged control positive group. Eighty percent in control positive non vaccinated challenged group and $20 \%$ in control negative non vaccinated challenged group.

\section{5-Result of histopathological examination \\ 5.1. Histopathological scoring of the intestinal parameters and pathological alteration lesions of jejunum and cecum within the different treated groups.}

The histopathological score as illustrated in the table

4. Pathological alteration lesions (Figure 6) were recorded in groups G2, G1 (control negative and control positive vaccinated groups). The jejunum of chicken supplemented with basal diet and vaccinated with the live attenuated coccidial vaccine (G2) showed the feature of catarrhal enteritis associated with mucosal lining degeneration, goblet cell proliferation and marked lymphocytic cells infiltration. The jejunum of chicken supplemented with mycotoxin-contaminated ration and vaccinated with the same vaccine (G1) revealed marked aggravation the inflammatory grade to reach to some cases to necrotic enteritis accompanied with focal ulceration of the lining mucosa. Also, a decrease in the intestinal morphometric parameters in comparison with previous group. Most of the jejunal villi showed marked blunting associated with decrease their length and decrease the crypt depth. $(\mathrm{p}<0.05)$. The jejunum of chicken supplemented with basal diet then challenged Showed mild degree of necrotic enteritis. While challenged birds subjected to mycotoxicated-diet revealed a marked degree of necrotic enteritis associated with necrosis, sloughing of mucosal lining and necrotic core. There was also a remarkable decrease in intestinal parameters.

Vaccination of the challenged birds showed improvement in intestinal parameters. While mycotoxin supplementation in diet of diseased birds with previous vaccination demonstrated marked retardation of jejunal morphometric parameters.

The cecum showed more prominent lesions than other intestinal sections (Figure 7) including the jejunum. The bird vaccinated with live coccidial vaccine (G2) showed a mild degree of necrotic typhlitis associated with the presence of different coccidial stages within the mucosal cell lining. The chicken kept on mycotoxins contaminated ratio and vaccinated with the same vaccine (G1) revealed a marked degree of necrotic enteritis, typhlitis accompanied by interstitial hemorrhage with a high number of different coccidial stages.

5.2. Histopathological findings of bursa of Fabricius and kidney in control negative and mycotoxicated control positive groups.

In Figure 8, the bursa of Fabricius in control negative bird showed normal bursal compartments with an increase of lymphoid elements (A) while the bursa of control positive birds showed separated follicles, edematous background and marked germinal centers necrosis associated with endodermal hyperplasia (B). The kidney of control negative bird revealed mild renal tubular degeneration mostly of granular eosinophilic cell swelling of the renal tubular epithelium (C) compared to kidney of control positive birds showed marked tubular degeneration accompanied with marked vacuolation of the renal tubules and interstitial inflammatory reaction mostly mononuclear cells (D). Later on, liver of control negative bird showed normal hepatic tissues (E) compared to liver of control positive birds showed hepatic degenerative changes represented by marked hydropic degeneration to multifocal hepatic necrosis associated with marked lymphocytic cells infiltration (F).

Table 1. The effect mycotoxin-contaminated feed on body weight of broiler chickens in vaccinated and non-vaccinated groups against coccidiosis.

\begin{tabular}{lccccc}
\hline Groups & Week 1 & Week 2 & Week 3 & $\begin{array}{c}\text { Overall } \\
\text { Mean } \pm \text { SE }\end{array}$ \\
\hline G1 (mycotoxicated, vaccinated group) & $128.00 \pm 5.28$ & $266.00 \pm 10.92$ & $466.50 \pm 19.90$ & $697.00 \pm 25.64$ & $389.38 \pm 35.35^{\mathrm{b}}$ \\
G2 (Non-mycotoxicated, vaccinated group) & $133.50 \pm 4.15$ & $275.50 \pm 5.60$ & $567.50 \pm 13.48$ & $883.00 \pm 40.76$ & $464.88 \pm 47.24^{\mathrm{a}}$ \\
G3 (mycotoxicated, non-vaccinated group) & $110.00 \pm 6.32$ & $282.50 \pm 20.87$ & $487.00 \pm 25.65$ & $628.50 \pm 23.71$ & $377.00 \pm 33.09^{\mathrm{b}}$ \\
G4 (Non-mycotoxicated, non-vaccinated group) & $116.00 \pm 7.59$ & $303.00 \pm 14.32$ & $578.00 \pm 34.27$ & $748.50 \pm 25.85$ & $436.38 \pm 40.58^{\mathrm{a}}$ \\
\hline
\end{tabular}

${ }^{\mathrm{a}, \mathrm{b}}$ Different superscripts indicate significant difference at $p<0.05$; SE: Standard error 
Elnabarawy et al., 2020

Table 2. The effect mycotoxin-contaminated feed on Eimeria oocysts count of broiler chickens in vaccinated and non-vaccinated groups against coccidiosis

\begin{tabular}{|c|c|c|c|c|c|}
\hline Groups & Time 1 & Time 2 & Time 3 & Time 4 & $\begin{array}{c}\text { Overall Mean } \pm \text { SE of } \\
\text { times/group }\end{array}$ \\
\hline G1 (mycotoxicated \&vaccinated group ) & $4.6 \times 10^{6} \pm 6.7 \times 10^{5}$ & $3.5 \times 10^{6} \pm 1.7 \times 10^{5}$ & $2.8 \times 10^{6} \pm 5.9 \times 10^{4}$ & $3.9 \times 10^{5} \pm 5.8 \times 10^{4}$ & $2.8 \times 10^{6} \pm 4.9 \times 10^{5 \mathrm{a}}$ \\
\hline G3 (mycotoxicated, non-vaccinated group) & $0.0 \pm 0.0$ & $0.0 \pm 0.0$ & $0.0 \pm 0.0$ & $0.0 \pm 0.0$ & $0.0 \pm 0.0$ \\
\hline $\mathrm{p}$-value & & & & & $<0.0001$ \\
\hline
\end{tabular}

Table 3. The effect mycotoxin-contaminated feed on Eimeria oocysts count of broiler chickens in vaccinated with anticoccidial vaccine and non-vaccinated groups post-challenge with Eimera tenella oocysts $\left(5 \times 10^{4} / \mathrm{chick}\right) 14$ days post vaccination.

\begin{tabular}{lccc}
\hline Groups & Time 1 & Time 2 & $\begin{array}{c}\text { Overall Mean } \pm \text { SE of } \\
\text { times/group }\end{array}$ \\
\hline G1 (mycotoxicated \&vaccinated group) & $3.7 \times 10^{6} \pm 2.4 \times 10^{5}$ & $4.9 \times 10^{5} \pm 5.8 \times 10^{4}$ & $2.1 \times 10^{6} \pm 7.2 \times 10^{5 \mathrm{a}}$ \\
G2 (Non-mycotoxicated, vaccinated group) & $1.9 \times 10^{6} \pm 1.2 \times 10^{5}$ & $1.6 \times 10^{5} \pm 2.0 \times 10^{4}$ & $1.0 \times 10^{6} \pm 3.9 \times 10^{5 \mathrm{~b}}$ \\
G3 (mycotoxicated, non-vaccinated group & $3.5 \times 10^{6} \pm 2.3 \times 10^{4}$ & $8.5 \times 10^{5} \pm 1.8 \times 10^{5}$ & $2.2 \times 10^{6} \pm 5.9 \times 10^{5 \mathrm{a}}$ \\
G4 (Non-mycotoxicated, non-vaccinated group) & $2.9 \times 10^{6} \pm 4.8 \times 10^{5}$ & $7.1 \times 10^{5} \pm 5.8 \times 10^{4}$ & $1.8 \times 10^{6} \pm 5.5 \times 10^{5 \mathrm{a}}$ \\
\hline
\end{tabular}

$\overline{\mathrm{a}, \mathrm{b}}$ Different superscripts indicate significant difference at $p<0.05$

Table 4. Histopathological scoring of the intestinal parameters (jejunum and cecum) within the different groups of broiler chickens

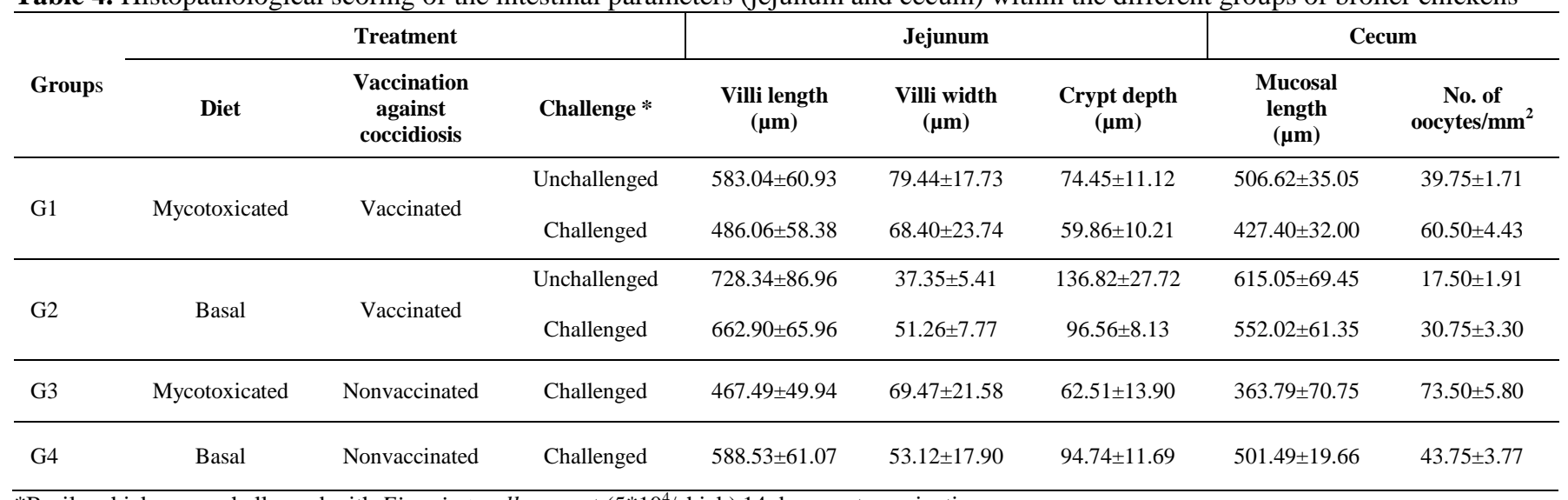

*Broiler chicks were challenged with Eimeria tenella oocyst (5*104/chick) 14 days post-vaccination. 

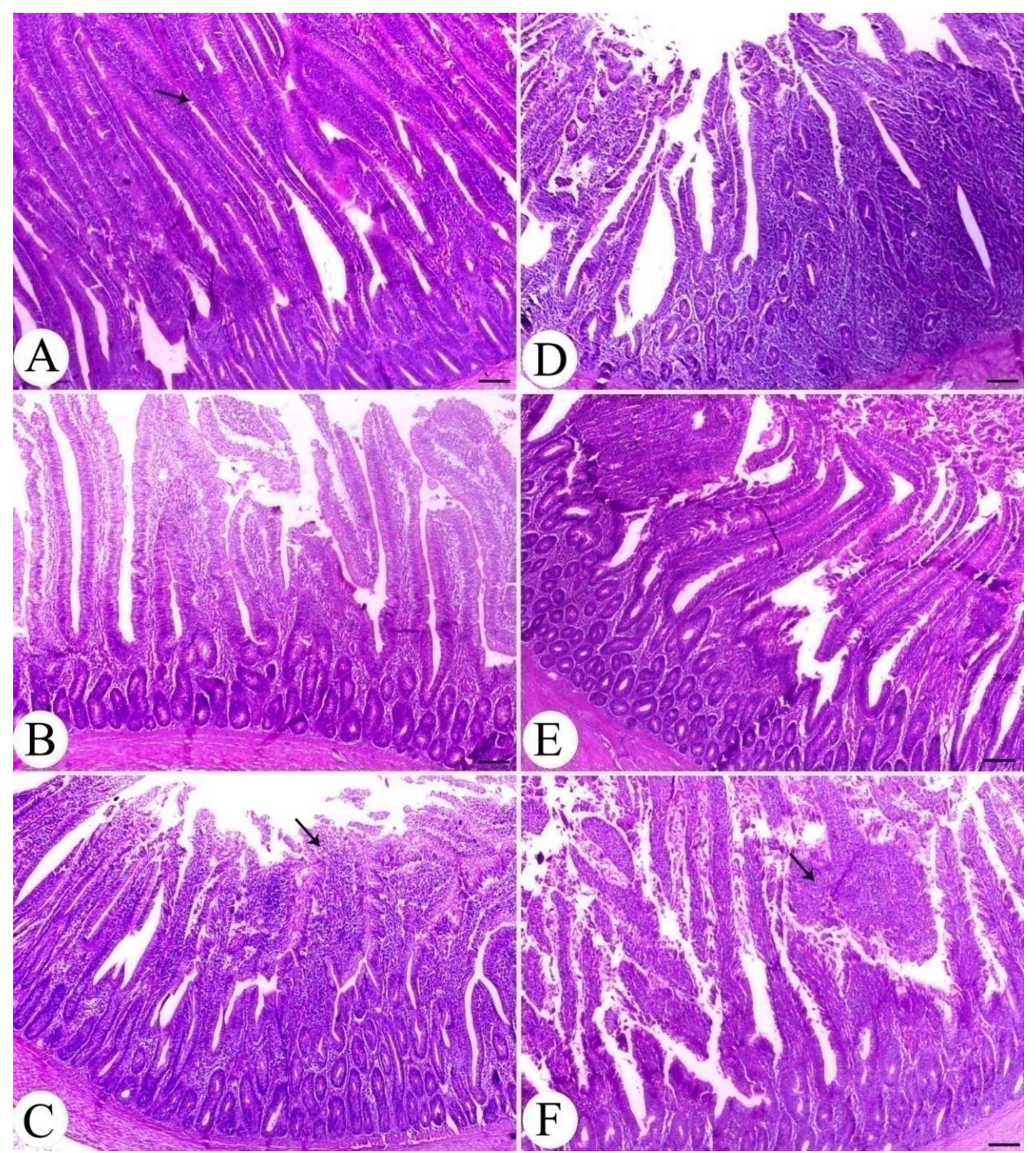

Figure 6. Intestine (jejunum section) of different chicken groups ( $2^{\text {nd }}$ week); A) chicken supplemented with basal diet and vaccinated with live anti-coccidial vaccine showing normal intestinal villi (arrow indicates normal mucosal lining); B) chicken supplemented with mycotoxin-contaminated ration and vaccinated with the same vaccine showing blunting of the intestinal villi and decrease of their length; C) chicken supplemented with basal diet and then challenged with E. tenella oocysts revealing marked degenerative changes within the covering mucosa (arrow); D) chicken supplemented with mycotoxin-contaminated ration and challenged showing severe catarrhal enteritis (arrow indicates marked inflammatory cells infiltration mostly mononuclear cells); E) chicken supplemented with normal ration, vaccinated and challenged showing decrease the degenerative and desquamative changes and with improvement of villi length; F) chicken supplemented with mycotoxin-contaminated ration, vaccinated and challenged showing necrosis and sloughing of the mucosal lining (arrow). H\&E, X200. 


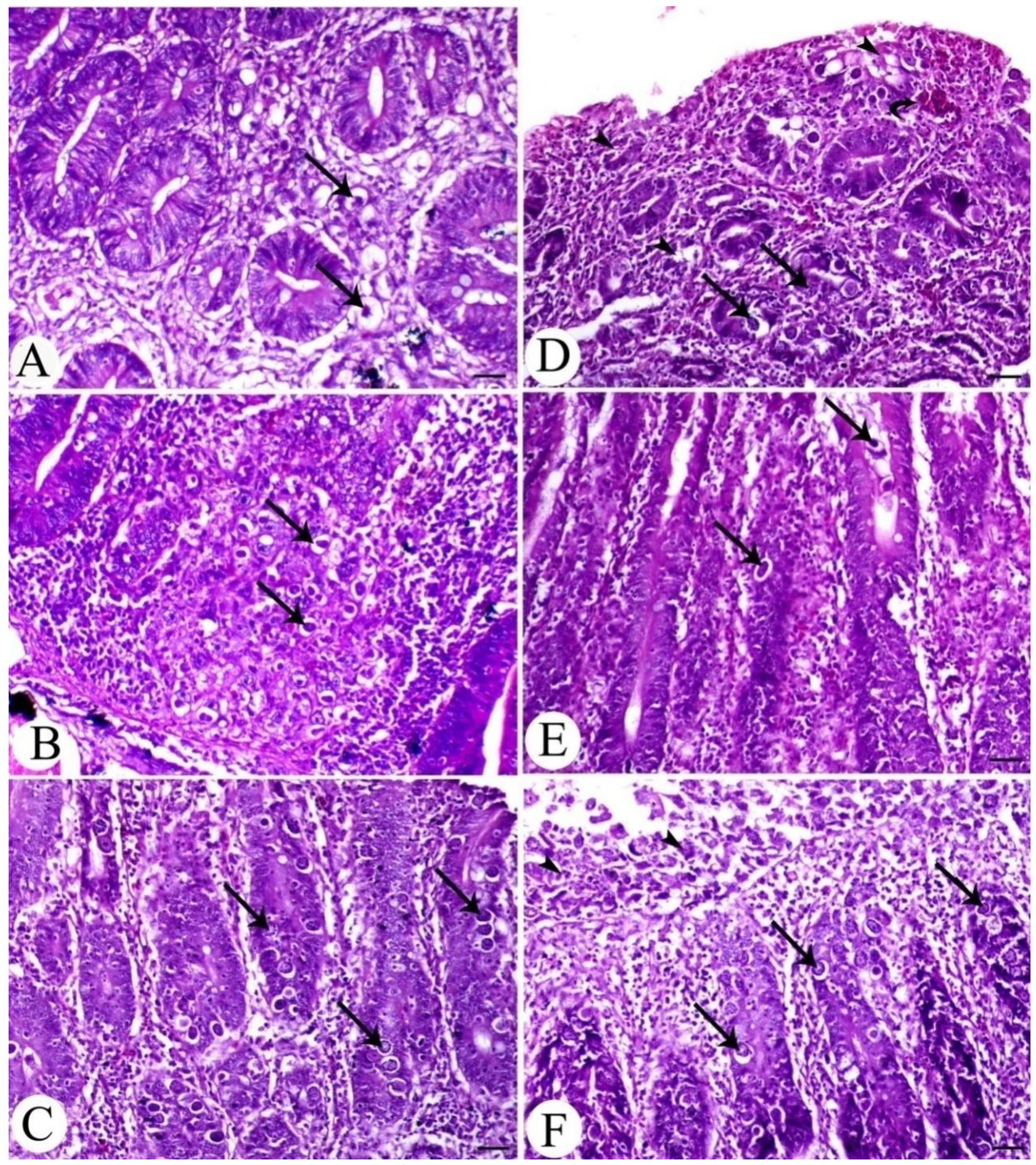

Figure 7. Cecal section of different groups ( $2^{\text {nd }}$ week); A) chicken supplemented with basal diet and vaccinated with live anticoccidial vaccine showing presence of few numbers of coccidial oocysts (arrow) with slight intestinal crypt degeneration; B) chicken supplemented with mycotoxins-contaminated ration and vaccinated with the same vaccine showing presence of remarkable number of parasitic oocysts within the interstitial tissue and glandular epithelium mucosa (arrows); C) chicken supplemented with basal diet and then challenged revealing marked degenerative and hyperplastic changes within the crypt epithelium associated with presence of the different coccidial stages within the epithelium (arrows); D) chicken supplemented with mycotoxins-contaminated ration and challenged showing severee necrotic typhilitis (arrowheads indicates necrosis of the intestinal crypts) and marked interstitial hemorrhage (curved-arrow) associated with coccidial stages (arrows); E) chicken supplemented with normal ration, vaccinated and challenged showing a marked decrease of coccidial stages with the intestinal mucosa (arrows) and with subsequent decrease intestinal degeneration and necrosis; F) chicken supplemented with mycotoxin-contaminated ration, vaccinated and challenged showing superficial sloughing of the mucosal lining (arrowheads) and crypt necrosis accompanied with coccidial parasites (arrows). H\&E, X200. 

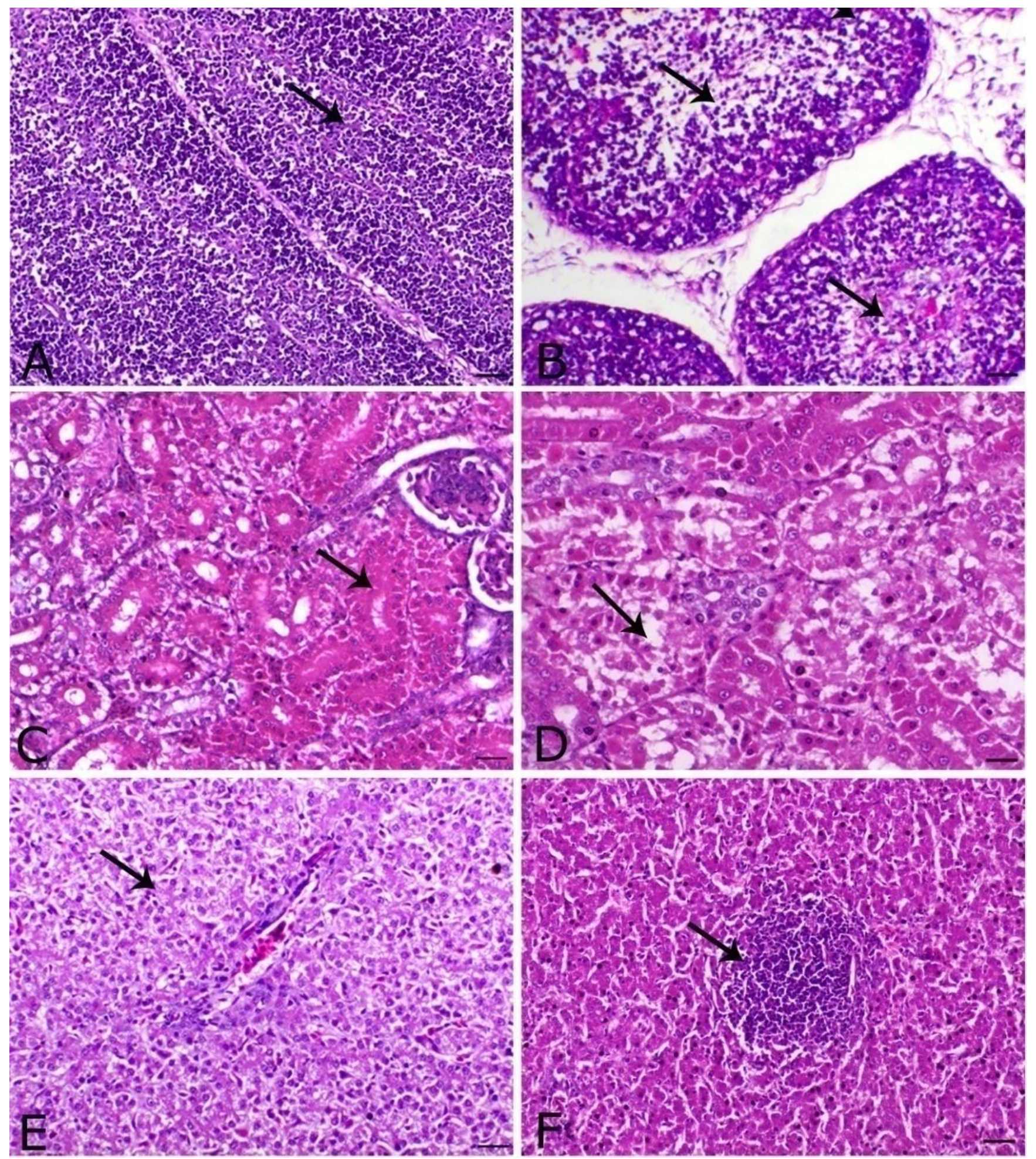

Figure 8. The bursal, renal and hepatic lesions in control negative and positive group according to mycotoxins supplementation. A represents the bursa of Fabricius in control negative bird showing mild reactive lymphoid hyperplasia (arrow); B) the bursa of control positive birds that showing marked lymphoid depletion of the germinal centers (arrow); C) kidney of control negative bird that showing mild renal tubular degeneration (arrow indicates granular eosinophilic cell swelling); D) the kidney of control positive birds that showing marked tubular degeneration (arrow); E) liver of control negative bird that showing mild hepatic vacuolation (arrow); F) the liver of control positive birds that showing focal hepatic necrosis associated with marked lymphocytic cells infiltration (arrow). H\&E, 


\section{DISCUSSION}

Avian mycotoxicosis is a great constraint in the poultry industry due to the development of immunosuppression, hepatotoxicity, and nephrotoxicity. Mycotoxins can transfer through chicken meat and egg to human, therefore, avian mycotoxicosis also is considered a public health issue (Adeniran et al., 2013).

The obtained result revealed that a significant reduction in body weight of groups 1 and 3 in comparison to the other groups. In the same respect, Aravind et al. (2003) stated that naturally mycotoxin contaminated feed at starter and growing period can affect broiler growth performance. Moreover, Girish and Smith (2008) and Yang et al. (2012) recorded a reduction in feed consumption and nutrient digestibility. They referred to these effects due to alterations caused by mycotoxins on intestinal morphology. Rosa and Santurio (2005); EFSA (2009) and Resanovic et al. (2009) reported that the most common clinical signs of mycotoxins in broiler chickens are reduced feed intake and weight gain, poor food conversation ratio, increase mortality, reduced immune response, organ damage, meat discoloration, and skeletal abnormalities as tibial dyschondroplasia, articular gout. In the same respect, Andretta et al. (2011) stated that the mycotoxins presence in diets reduces weight gain by $14 \%$ when compared with the control groups.

The effect of different mycotoxins in gut health was reported by Liew and Redzwan (2018) as they described the different actions of aflatoxin, ochratoxin, and zearalenone and they include growth retardation, immunosuppression, and genotoxicity. They also revealed gut changes due to previously mentioned mycotoxins and those are alterations in nutrient absorption, inhibition of cell growth, increase lactate dehydrogenase activity and caused genetic damages that mean disruption of intestinal barrier, cell proliferation as the development of subepithelial space and villi degeneration, cell apoptosis a1nd immune system.

Mycotoxins are potent immune suppressive factors and produced a negative effect on both humoral and cellmediated immune response to live New Castle disease viral vaccine resulting in pronounced lowering in protection rate against infection with VVND (viscerotropic velogenic New Castle disease virus). These aforementioned results were obtained during experimental work carried out by Anwaar et al. (2016).

Oswald et al. (2006) stated that mycotoxins ingestion impaired the acquired immunity through vaccination and multi contamination with mycotoxins altered immunemediated components. In addition, immunosuppression induced by mycotoxins causing a decrease in host resistance and consequently increase susceptibility to infectious diseases and reduce vaccine efficacy.

While Pier (1992) confirmed that the vaccinal immunity in properly vaccinated flocks is broken down due to the contamination of mycotoxins in feed.

In the same respect, Desjardins (2006) found that Fusarium mycotoxins are affecting different cellular and molecular levels those resulting in adverse effect on proliferation and differentiation of immune system cells.

The explanation of that mycotoxins are immune suppressive resulting in inhibition of protein synthesis or impairment of the activity or secretory functions of immune system cells as well as synthesis of cytokines that regulate the communication network of the immune system (Swamy et al., 2004; Oswald et al., 2006). Moreover, Oswald et al. (2006) reported that in vitro phagocytosis, intracellular killers were inhibited by aflatoxin B1. Gastrointestinal tract (GIT) function is feed ingestion, digestion, energy, and nutrients absorption, as well as elimination of waste products (Celi et al., 2017). Epithelial layer is the inner most of intestinal mucosa of vital importance. As they contains enteroendocrines, enterocytes and gablet cells at villi whereas the paneth cells, located under the crypts (Fink and Koa, 2016). This epithelium layer working as normal barrier to prevent the entry of pathogens and toxins moreover, it is the site for nutrient absorption including electrocytes (Constantinescu and chon, 2016). Desmosomes tight junctions and adherent junctions are connecting intestinal epithelial cells. These junctions controlling the intercellular space and regulate selective paracellular ionic solute transport (Capaldo et al., 2014). Zearalenone well recognized to be implicated in reproductive disorders. Zhou et al. (2017) indicated that zearalenone has hepatotoxic, hematotoxic, immunotoxic and genotoxic effect. The effect of zearalenone on GIT is that, inducing cell death without affecting cell integrity (Marin et al., 2015). Ochratoxin the immunsuppresive, teratogenic and nephrotoxic substance reflected faster and more harmful parasite infection induced by E. acervulina and E. adenoides in OTA treated chicks and turkeys (Laderia et al., 2017). As Manafi et al. (2011) indicated that high lesion and oocyst indices in the intestine due to Eimeria infection caused more damage for mucosa and this is attributed to increasing intestinal permeability (McLaughlin et al., 2004). Solcan et al. (2015) reported that OTA fed broilers caused a decrease in villi height and 
increase apoptosis of intestinal epithelial cells. Numerous studies on broilers fed with aflatoxin B contaminated diet showed that reduction in the density (weight/ length) of intestine (Hossein and Gurbuz, 2015). Moreover, the increased apoptosis was corresponded to lower jejuneal villi height (Peng et al., 2014).

\section{DECLARATION}

\section{Acknowledgment}

This study was financially supported by scientific research sector of Cairo University through the project titled "Mycotoxicosis, the natural potent immunosuppressive carcinogen of veterinary and public health concern".

\section{Authors' contributions}

Anwaar M. Elnabarawy designed the experiment, provided facilities and material needed, performed mycotoxin detection and determination, collected results, and wrote and revised the manuscript. Marwa M. Khalifa prepared the challenging doses of Eimeria oocysts, collected dropping samples and counted numbers of shaded oocysts in at least 40 samples, contributed to manuscript writing. Khaled S. Shaban recorded the body weight, daily observation for clinical symptoms, mortality and contributed to detection and determination of mycotoxins levels in feed. Walied S. Kotb prepared and examined histopathological sections.

\section{REFERENCES}

Adeniran, LA, Peter OA, Abdulazeez SN and Olatunde OH (2013). Avian mycotoxicosis in developing countries. In: Mycotoxin and food safety in developing countries. Makun, H.A. Ed. In Tech. DOI: http://dx.doi.org/10.5772/3414.

AE, Ed. Fusarium Mycotoxins Chemistry, Genetics and Biology.Minnesota: APS, pp. 53-54.

Andretta I, Kipper M, Lehnen CR, Hauschild, L Vale MM and Lovatto PA (2011). Meta-analytical study on productive and nutritional interactions of mycotoxins in broilers. Poultry Science, 90: 19341940. DOI: https://dx.doi.org/10.3382/ ps.2011-01470.

Anwaar ME, Madian K, Aly EA and Madbouly Y (2016). The effect of mycotoxins on immune response of broilers to the live NC vaccines applied by different routes. Middle East Journal of Applied Sciences, 6 (1):51-58. Available at: http://www.curresweb.com/mejas/mejas/2016/51-58.pdf

AOAC (1995). Association of Official Analytical Chemists, Official Methods of Analysis. 16th ed. Method 991.31. Gaithersburg, MD, USA. Available

http://onlinelibrary.wiley.com/doi/10.1111/j.17503841.2010.01980.x/pdf

Aravind KK, Patil VS, Devegowda G, Umakantha B and Ganpule SP (2003). Efficacy of esterified glucomannan to counteract mycotoxicosis in naturally contaminated feed on performance and serum biochemical and hematological parameters in broilers. Poultry Science, 82:571-576. DOI: https://dx.doi.org/10.1093/ps/82.4.571.

Bancroft JD, Stevens A and Turner DR (1996). Theory and practice of Histological Technique. Fourth Ed., Churchill Livingstone, New York, London, San Francisco, Tokyo. Available at: https://trove.nla.gov.au/work/10963990

Capaldo CT, Farkas AE and Nusrat A (2014). Epithelial adhesive junctions. F1000Prime Report, 6:13. DOI: https://doi: 10.12703/ P6-1.

Celi P, Cowieson A, Fru-Nji F, Steinert R, Kluenter AM and Verlhac V (2017). Gastrointestinal functionality in animal nutrition and health: new opportunities for sustainable animal production. Animal Feed Science and Technology, 234: 88-100. DOI: https:// 10.1016/ j.anifeedsci. 2017. 09.012.

Collier CT, Hofacre CL, Payne AM, Anderson DB, Kaiser P, Mackie RI, and Gaskins HR (2008). Coccidia-induced mucogenesis promotes the onset of necrotic enteritis by supporting Clostridium perfringens growth. Veterinary Immunology and Immunopathology, 122:104115. DOI: https://doi.org/10.1016/j.vetimm.2007.10.014.

Constantinescu CS and Chou IJ (2016). Intestinal bacterial antigens, toxin induced pathogenesis and immune cross-reactivity in neuromyelitis optica and multiple sclerosis, in Neuro-ImmunoGastroenterology, eds C. S. Constantinescu R, Arsenescu and V. Arsenescu (Cham: Springer), 227-236. DOI: https:// 10.1007/978-3319-28609-9-13.

Dahiya JP, Wilkie DC, Van Kessel AG and Drew MD (2006). Potential strategies for controlling necrotic enteritis in broiler chickens in post-antibiotic era. Animal Feed Science and Technology, 129:60 88 DOI: https://doi.org/10.1016/j.anifeedsci.2005.12.003.

Dalloul RA, and Lillehoj HS (2006). Poultry coccidiosis: Recent advancements in control measures and vaccine development. Expert Review of Vaccines 5:143-163. DOI: https://doi.org/10.1586/14760584.5.1.143.

Desjardins AE (2006). Mechanism of action of trichothecenes. In: Desjardins

European Feed Safety Agency (EFSA) (2009). Review of mycotoxindetoxifying agents used as feed additives: mode of action, efficacy and feed food safety. Available at: http://www.efsa.europa.eu/en/supporting/pub/22e.htm.

FAO, Food and Agriculture Organization of the United Nations (2003). Manuals of Food Quality Control 10. Training in Mycotoxins Analysis.

Fink J and Koo BK (2016). Clonal evolution of stem cells in the gastrointestinal tract. Advances in Experimental Medicine and Biology: Stem Cells, Pre-neoplasia, and Early Cancer of the Upper Gastrointestinal Tract, eds M. Jansen and N. Wright (Cham: Springer), 11-25. DOI: https:// 10.1007/978-3-319-41388-4-2

Forgacs J and Carll WT (1955). Preliminary mycotoxic studies on hemorrhagic diseases in poultry. Veterinary Medicine, 50: 172.

Girish CK and Smith TK (2008). Effects of feeding blends of grains naturally contaminated with Fusarium mycotoxins on small intestinal morphology of turkeys. Poultry Science, 87: 10751082. DOI: https://doi: 10.3382/ ps.2007-00379.

Hossein A, and Gurbuz Y (2015). Aflatoxins in poultry nutrition.

Kahramanmara s s Sütçü Imam Üniversitesi Doga Bilimleri Dergisi, 18, 1 5. DOI: https://doi: 10.18016/ksujns.98227.

Karaman M, Basmacioglu H, Ortatatli M, Oguz Dr H (2005). Evaluation of the detoxifying effect of yeast glucomannan on aflatoxicosis in broilers as assessed by gross examination and histopathology. Brit Poultry Science, 46:394-400. DOI: https:// doi.org/10.1080/00071660500124487. 
Khaier MA, Abdelhalim AI and Abukashawa SM (2015). Isolation and Morphological Identification of Eimeria tenella (Family:Eimeriiddae) from Khartoum State (Sudan). Journal of Applied and Industrial Sciences, 3:177-181. Available at: https:// www. researchgate. net/ publication/296486011.

Kubena LF, Huff WE, Harvey RB, Corrier DE, Phillips TD, and Creger CR (1988). Influence of ochratoxin A and deoxynivalenol on growing broiler chicks. Poultry Science, 67:253-260. DOI:https://doi.org/10.3382/ps.0670253.

Ladeira C, Frazzoli C and Orisakwe OE (2017). Engaging one health for noncommunicable diseases in Africa: perspective for mycotoxins. Frontiers in Public Health, 5:266. DOI: https://doi: 10.3389/ fpubh. 2017.00266.

Liew WP, Mohd-Redzwan S (2018): Mycotoxin: its impact on gut health and microbiota. Frontiers in Cellular and Infection Microbiology, 8, 1-17, DOI: https://doi:10.3389/fcimb.2018.00060.

Manafi M, Mohan K and Ali MN (2011). Effect of ochratoxin A on coccidiosis-challenged broiler chicks. World Mycotoxin Journal, 4:177-181. DOI:https://doi: 10.3920/ WMJ2010.1234.

Marin DE, MotiuM., and Taranu I (2015). Food contaminant zearalenone and its metabolites affect cytokine synthesis and intestinal epithelial integrity of porcine cells. Toxins,7: 1979-1988. DOI: https:// doi: 10.3390/toxins 7061979 .

McDougald LR and Fitz Coy SH (2013). Coccidiosis.In D.E. Swayne, J.R. Glisson, L.R. McDougald, L.K. N olan, D.L. Suarez \& V.L. Nair (Eds.), Diseases of Poultry 13th edn (pp. 1148 1166). Available at: https://www.wiley.com/enae/Diseases+of+Poultry,+13th+Edition-p-9781118719732

McLaughlin J, Padfield PJ, Burt, JP, and O'Neill CA (2004). Ochratoxin A increases permeability through tight junctions by removal of specific claudin isoforms. American Journal of Physiology Cell Physiology, 287: C1412-C1417. https://doi:10.1152/ajpcell.00007.2004.

Oswald IP, Marin DE, Bouhet S, Pinton P, Taranu I, Accensi F (2006). Immunotoxicological risk of mycotoxins for domestic animals. Food Additives and Contaminants, 22:354e60. DOI:https:// doi :10.1080/02652030500058320.

Peng X, Zhang S, Fang J, Cui H, Zuo Z and Deng J (2014). Protective roles of sodium selenite against aflatoxin B1-induced apoptosis of jejunum in broilers. International Journal of Environmental Research and Public Health, 1: 13130-13143. DOI:https:// doi: 10.3390/ijerph111213130.

Pier AC (1992). Major biological consequences of aflatoxicosis in animal production. Journal of Animal Science, 70: 8860-8867. DOI:https:// doi: $10.2527 / 1992.70123964 x$.

Resanovic RM, Nesik KD, Nesic VD, Palic TD and Jacevic VM (2009). Mycotoxins on poultry production. Proc. Nat.Sci, Matica Srpska Novi Sad, 116:7-14.DOI: https://doi:10.2298/ZMSPN0916007R.
Rosa AP and Santurio J (2005). Mycotoxins on poultry production. In : Veterinary poultry association. The $14^{\text {th }}$ world veterinary poultry congress. Istanbul, Turkey. 126-137. Available at: http://www.vtd.org.tr/siteimages/meeting7/17-rosa-

mycotoxins_on_poultry_production.pdf

Shirley MW, Bushell AC, Bushell JE, McDonald V and Roberts BA (1995). Live attenuated vaccine for control of avian coccidiosis: trials in broiler breeders and replacement layer flocks in the United Kingdom. Veterinary Record, 137:453-457.DOI: https://doi:10.1136/vr.137.18.453.

Shirley MW, Smith AL and Blake DP (2007) Challenges in the successful control of the avian coccidia. Vaccine 25:5540-5547. DOI:https:// doi:10.1016/j.vaccine.2006.12.030

Solcan C, Pavel G, Floristean V, Chiriac I, Slencu B and Solcan G (2015). Effect of ochratoxin A on the intestinal mucosa and mucosa associated lymphoid tissues in broiler chickens. Acta Veterinaria Hungarica, 63:30-48. DOI:https://doi: 10.1556/ AVet.2015.004.

Soulsby E (1982). Helminths, Arthropods and Protozoans of Domesticated Animals. 7 th Ed., Bailliere Tindall, London, UK. Available at: https://www.worldcat.org/title/helminths-arthropodsand-protozoa-of-domesticated-animals/oclc/855675040

Swamy HV, Smith TK, Karrow NA, Boermans HJ (2004). Effects of feeding blends of grains naturally contaminated with Fusarium mycotoxins on growth and immunological parameters of broiler chickens. Poultry Science 83:533-543. DOI: https://doi:10.1093/ps/83.4.533.

Trenholm HL, Prelusky DB, Young JC and Miller JD (1989). A practical guide to the prevention of Fusarium mycotoxins in grain and animal feedstuffs. Archives of Environmental Contamination and Toxicology, 18:443-451. Available at: https://link.springer.com/article/10.1007\%252FBF01062372

Velkers FC, Blake DP, Graat EA, Vernooij JC, Bouma A, Jong MC and Stegeman JA (2010). Quantification of Eimeria acervulina in faeces of broilers: comparison of McMaster oocyst counts from 24h faecal collections and single droppings to real-time PCR from cloacal swabs. Veterinary Parasitology, 169: 1-7. DOI: https://doi:10.1016/j.vetpar.2010.01.001.

Yang C, Lates V, Prieto-Simon B, Marty J, and Yang X. (2012). Aptamer-DNAzyme hairpins for biosensing of ochratoxin A. Biosensors and Bioelectronics, 32, 208-212. DOI:https:// doi: 10.1016/j.bios.2011.12.011.

Zhou H, George S, Hay C, Lee J, Qian H and Sun X (2017). Individual and combined effects of aflatoxin B 1, deoxynivalenol and zearalenone on HepG2 and RAW 264.7 cell lines. Food and Chemical Toxicology, 103, 18-27. DOI:https://doi: 10.1016/j.fct.2017.02.017. 